\title{
Image Contrast Enhancement Based on a Histogram Transformation of Local Standard Deviation
}

\author{
Dah-Chung Chang* and Wen-Rong Wu, Member, IEEE
}

\begin{abstract}
The adaptive contrast enhancement (ACE) algorithm, which uses contrast gains (CG's) to adjust the highfrequency components of images, is a well-known technique for medical image processing. Conventionally, the CG is either a constant or inversely proportional to the local standard deviation (LSD). However, it is known that conventional approaches entail noise overenhancement and ringing artifacts. In this paper, we present a new ACE algorithm that eliminates these problems. First, a mathematical model for the LSD distribution is proposed by extending Hunt's image model. Then, the CG is formulated as a function of the LSD. The function, which is nonlinear, is determined by the transformation between the LSD histogram and a desired LSD distribution. Using our formulation, it can be shown that conventional ACE's use linear functions to compute the new CG's. It is the proposed nonlinear function that produces an adequate CG resulting in little noise overenhancement and fewer ringing artifacts. Finally, simulations using some X-ray images are provided to demonstrate the effectiveness of our new algorithm.
\end{abstract}

Index Terms - Adaptive contrast enhancement, histogram transformation, local standard deviation (LSD), radiography.

\section{INTRODUCTION}

$\mathbf{I}$ MAGE contrast enhancement is important in medical applications. This is due to the fact that visual examination of medical images is essential in the diagnosis of many diseases. In applications such as chest radiography and mammography [1], [2], the image contrast is inherently low due to the small differences in the X-ray attenuation coefficients. The problem is further complicated if an image consists of several regions with different X-ray attenuation characteristics. For example, in chest radiography, the mediastinum and the lung field have different exposures. It is usually desirable to enhance the details in both regions simultaneously. Thus, a considerable amount of research has focused on this subject. The development of enhancement algorithms is based on some visual principles. It is known that the human eye is sensitive to high-frequency signals. Although details usually correspond to high-frequency signals, their visibility becomes low when they are embedded in strong low-frequency background signals. Thus, properly amplifying the high-frequency components will improve visual perception and help diagnosis.

Manuscript received February 6, 1997; revised June 15, 1998. The Associate Editor responsible for coordinating the review of this paper and recommending its publication was C. Roux. Asterisk indicates corresponding author.

*D.-C. Chang is with the Department of Communication Engineering, National Chiao Tung University, Hsinchu, Taiwan, R.O.C. (e-mail: wrwu@cc.nctu.edu.tw).

W. -R. Wu is with the Department of Communication Engineering, National Chiao Tung University, Hsinchu, Taiwan, R.O.C.

Publisher Item Identifier S 0278-0062(98)08579-6.
Linear contrast stretching and histogram equalization are two widely utilized methods for global image enhancement [4]-[7]. The former linearly adjusts an image's dynamic range, and the latter uses the input-to-output mapping relation obtained from the integral of the image histogram. Although these methods are simple, they do not take into account local details. In addition, global histogram equalization (GHE) has the undesired effect of overemphasizing noise [19].

In diagnostic medical images, local details may be more important than global contrast. Adaptive histogram equalization (AHE) [8]-[11] and adaptive contrast enhancement (ACE) [12]-[19] are two well-known local enhancement methods. AHE algorithms map the gray values of pixels using the relationships obtained from the local histograms. Although this improves image contrast, it requires intensive computations [8]. The bilinear interpolation technique was developed [9], [10] to reduce the computational burden. It first divides images into blocks, and then calculates the mapping functions of those blocks. To enhance a particular pixel, the mapping function is interpolated using four mapping functions associated with its four neighboring blocks. In this algorithm, the only parameter to be determined is the block size. To facilitate the visualization of local details, the block size must be small, however, small blocks increase computational requirements. Thus, there is a tradeoff between the enhancement of local details and computational loading when the interpolated AHE method is used.

The ACE algorithms adopt unsharp masking techniques. The details of which are explained as follows: an image is separated into two components; the low-frequency unsharp mask obtained by low-pass filtering of the image, and the high-frequency component obtained by subtracting the unsharp mask from the original image. The high-frequency component is then amplified and added back to the unsharp mask to form an enhanced image. The main concern in the ACE method is how to determine the CG. There are two conventional methods for calculating the CG. The simplest method is to use the gain as a constant [12]-[16]. Due to the low-pass characteristic of the unsharp mask, high-frequency components near the edges are strongly enhanced by this method which results in socalled "ringing artifacts." The other method uses the CG as an inverse proportional to the local standard deviation (LSD) [17], [18]. The CG, thus, becomes large for regions with low LSD values overenhancing background noise. Recently, Ji, Sundareshan, and Roehrig [19] proposed separating detail and smooth regions and using smaller gains for smooth regions. Relying on the local image contrast and the observer's just- 
noticeable-difference criterion, they developed an algorithm substantially reducing the problems.

Note that the ringing effect and the noise overenhancement are due to the use of very large CG's in regions with low spatial and high spatial activities. Details often correspond to the regions with medium spatial activities. Thus, if a $\mathrm{CG}$ can have larger values at the region with medium spatial activity and smaller values at regions with low and high spatial activities, ringing effects and noise overenhancement can be reduced. In this research, we developed a new ACE algorithm having these properties. First, we extended Hunt's image model [20], [21] and proposed a mathematical model for LSD distributions. The new CG was then formulated as a function of LSD. This function, which is nonlinear, was determined by the transformation between the LSD histogram and a desired LSD distribution. It can be shown that conventional ACE algorithms are special cases of our method, where the mapping functions between the new CG and LSD are linear. This is why the algorithm we formulated reduces the effects of ringing and noise enhancement. Finally, we applied the new algorithm to enhance some X-ray images for demonstrating its effectiveness.

This paper is organized as follows: Section II briefly reviews the conventional ACE approaches. Section III describes our LSD model. In Section IV, the new ACE algorithm is derived. We show the simulation results in Section V. Conclusions are drawn in Section VI.

\section{Conventional ACE Algorithms}

In this section, we review the conventional ACE algorithms. The unsharp mask corresponds to the low-frequency component of an image. For a particular pixel, this can be calculated by averaging the gray values of pixels over a local area centered at the pixel. Let $x(i, j)$ be the gray value of a pixel in an image. The local area is defined as a $(2 n+1) \times(2 n+1)$ window centered at $(i, j)$ where $n$ is an integer number. Note that the window's shape is not necessarily square. The local mean, i.e., the low-frequency component, of a pixel $(i, j)$ can be computed as

$$
m_{x}(i, j)=\frac{1}{(2 n+1)^{2}} \sum_{k=i-n}^{i+n} \sum_{l=j-n}^{j+n} x(k, l)
$$

and the local variance as

$$
\sigma_{x}^{2}(i, j)=\frac{1}{(2 n+1)^{2}} \sum_{k=i-n}^{i+n} \sum_{l=j-n}^{j+n}\left[x(k, l)-m_{x}(i, j)\right]^{2}
$$

The term $\sigma_{x}(i, j)$ is the LSD. Let $f(i, j)$ denote the enhanced value of $x(i, j)$. The ACE algorithm gives

$$
f(i, j)=m_{x}(i, j)+G(i, j)\left[x(i, j)-m_{x}(i, j)\right] .
$$

The function $G(i, j)$ is the CG. The CG is usually greater than one, so that the high-frequency component $\left[x(i, j)-m_{x}(i, j)\right]$ can be enhanced. Determination of $G(i, j)$ is the crucial step in the ACE algorithm.
The simplest process is to let $G(i, j)$ be a constant, say $C$ where $C>1$. Then, (3) becomes

$$
f(i, j)=m_{x}(i, j)+C\left[x(i, j)-m_{x}(i, j)\right] .
$$

Using this constant gain, all high-frequency components are amplified equally. As a consequence, those strong highfrequency components will suffer from overenhancement. An example is the ringing artifact in the vicinity of the edges. Therefore, a special deemphasizing procedure must be added to this algorithm [16]. For example, in chest radiography applications [15], [16], the chest image is usually composed of the mediastinum and the lung field. One way to reduce overenhancement is to use different gains for these anatomical fields. Another contrast enhancement application can be found in Lee's work [12]. For convenience, we refer to the algorithm in (4) as the CGT algorithm.

Another method, that uses LSD information, was presented in [17]

$$
f(i, j)=m_{x}(i, j)+\frac{D}{\sigma_{x}(i, j)}\left[x(i, j)-m_{x}(i, j)\right]
$$

where $D$ is a constant. In this case, the CG itself is spatially adaptive. It is inversely proportional to LSD. In edges or other areas having high spatial activities, the LSD will be large. The CG's in those areas are then small. Thus, no ringing will result. However, in smooth areas, the LSD will be small. Noise will be amplified due to the large CG. Still, this approach requires an additional procedure to limit the CG's. Hereafter, we refer to (5) as the IPLSD algorithm.

\section{A MODEL FOR IMAGE LSD DistRIBUTION}

\section{A. The New Formulation}

First, we rewrite the IPLSD algorithm in (5) as follows:

$$
f(i, j)=m_{x}(i, j)+D\left[\frac{x(i, j)-m_{x}(i, j)}{\sigma_{x}(i, j)}\right] .
$$

If we treat $x(i, j)$ as a random variable, $m_{x}(i, j)$ will be its mean and $\sigma_{x}(i, j)$ its standard deviation. Equation (6) then indicates that the LSD of the output image is normalized, in other words, $f(i, j)$ will have a standard deviation of $D$ for all $i$ and $j$. This is an interesting observation. The CGT algorithm in (4) can also be rewritten in a similar form

$$
f(i, j)=m_{x}(i, j)+C \sigma_{x}(i, j)\left[\frac{x(i, j)-m_{x}(i, j)}{\sigma_{x}(i, j)}\right] .
$$

We can generalize (6) and (7) resulting in a new class of ACE algorithms described as follows:

$$
f(i, j)=m_{x}(i, j)+K(i, j)\left[\frac{x(i, j)-m_{x}(i, j)}{\sigma_{x}(i, j)}\right] .
$$

To distinguish from the conventional contrast gain (CCG) $G(i, j)$, we call $K(i, j)$ the modified contrast gain (MCG). Like the CCG, $K(i, j)$ determines the output contrast. It can be easily seen that, if $K(i, j)<\sigma_{x}(i, j)$, the contrast is 


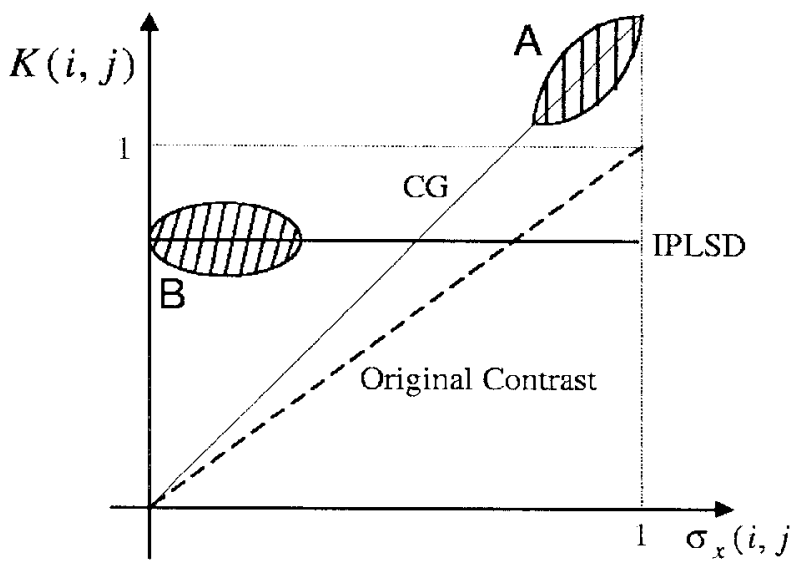

(a)

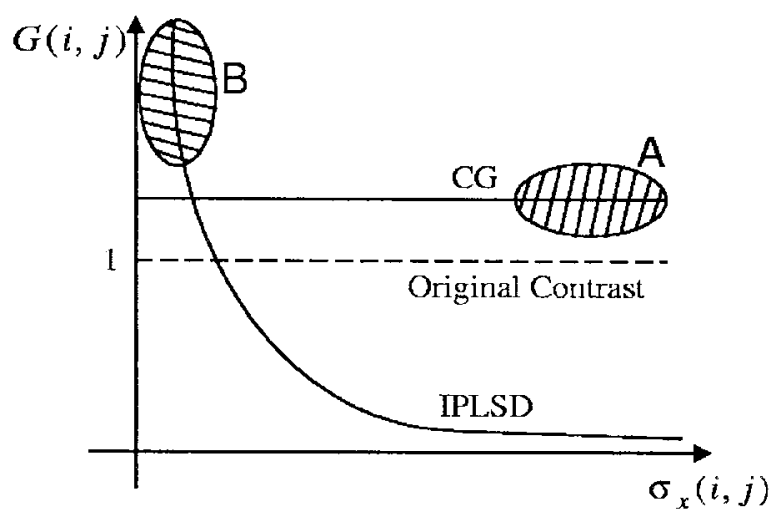

(b)

Fig. 1. Illustration of the causes of ringing artifacts and noise overenhancement in conventional ACE algorithms: (a) $K(i, j)$ versus $\sigma_{x}(i, j)$ and (b) $G(i, j)$ versus $\sigma_{x}(i, j)$.

reduced, if $K(i, j)=\sigma_{x}(i, j)$, the contrast remains the same, and if $K(i, j)>\sigma_{x}(i, j)$, the contrast is enhanced. Note that

$$
K(i, j)=D
$$

for the IPLSD algorithm and

$$
K(i, j)=C \sigma_{x}(i, j)
$$

for the CGT algorithm. Both are linear functions of $\sigma_{x}(i, j)$, and it is the linear functions that cause problems. We use Fig. 1 to illustrate this. In Fig. 1, we assume that $\sigma_{x}(i, j)$ has been normalized (divided by a maximum value). Fig. 1(a) gives $K(i, j)$ versus $\sigma_{x}(i, j)$ for the original contrast, the CGT algorithm, and the IPLSD algorithm; Fig. 1(b) gives $G(i, j)$ versus $\sigma_{x}(i, j)$. As we mentioned in Section I, details often correspond to regions with median $\sigma_{x}(i, j)$. Thus, if a constant gain for the CGT algorithm is adequate for detail regions, it will be too large for other regions with large $\sigma_{x}(i, j)$ (marked A in Fig. 1) and this causes the ringing artifacts. By contrast, if the gain function in the IPLSD algorithm is adequate for detail regions, it will be too large for regions with small $\sigma_{x}(i, j)$ (marked $\mathbf{B}$ in Fig. 1), in which noise overenhancement takes place. Also note that the IPLSD algorithm reduces the contrast in the high $\sigma_{x}(i, j)$ region. From Fig. 1(a), we can verify that the linear MCG is inadequate. If $K(i, j)$ can be a nonlinear function such that it is close to $\sigma_{x}(i, j)$ for low and high $\sigma_{x}(i, j)$ and larger than $\sigma_{x}(i, j)$ for median $\sigma_{x}(i, j)$, the contrast enhancement problems associated with conventional methods can be overcome. Apparently, $K(i, j)$ must be nonlinear.

Although the MCG in (8) can be a function of many variables, we only focus on the situation in which it is a function of $\sigma_{x}(i, j)$. In this situation, the MCG is reduced to a one-variable function denoted by $K(\cdot)$. From (8), we see that the LSD of $x(i, j)$ is $\sigma_{x}(i, j)$ and the LSD of $f(i, j)$, denoted by $\sigma_{f}(i, j)$, is $K\left(\sigma_{f}(i, j)\right)$. Thus, $K(\cdot)$ can be interpreted as a mapping function between $\sigma_{x}(i, j)$ and $\sigma_{f}(i, j)$, i.e.,

$$
\sigma_{f}(i, j)=K\left(\sigma_{x}(i, j)\right)
$$

Note that $\sigma_{x}(i, j)$ can be seen as a random variable having a certain histogram and $\sigma_{f}(i, j)$ as another random variable having its transformed histogram. If we have a desired LSD histogram, the function $K(\cdot)$ can then be calculated. This is to say that we can view the contrast enhancement as a histogram equalization (or transformation) problem. The only difference is that the input and output are LSD's instead of gray values. This is the foundation for our development of new ACE algorithms. Using this interpretation, we can see that the IPLSD algorithm transforms the LSD histogram into an impulse function [positioned at $\sigma_{f}(i, j)=D$ ] and the CGT algorithm linearly stretches the LSD histogram.

\section{B. A Model for the LSD Distribution}

The next problem is how to find the desired LSD histogram. We can always use the uniform distribution as that in histogram equalization. However, this is not appropriate since the LSD will not be uniformly distributed for most natural images. Below, we developed a model for LSD distributions. In [20], Hunt proposed a Gaussian image model given by

$$
x(i, j)=m(i, j)+r(i, j)
$$

where $m(i, j)$ is a nonstationary mean process and $r(i, j)$ is a stationary white Gaussian process. Using Hunt's model, we first consider the distribution of the variance estimate. Here, we assume that $m(i, j)$ is deterministic and slowly varying. As a consequence, $m(i, j)$ can be accurately estimated and ignored in the estimate of the variance. Let $\sigma_{0}^{2}$ be the true variance of $r(i, j)$ and $\zeta$ [instead of $\sigma_{r}^{2}(i, j)$ for simplicity] be the variance calculated from the $M+1$ samples of $r(i, j)$ in the local window. From mathematical statistics [22], we find that

$$
\frac{M \zeta}{\sigma_{0}^{2}} \sim \chi^{2}(M)
$$

where $\chi^{2}(M)$ is the chi-square distribution with $M$ degrees of freedom. For a large $M, \chi^{2}(M)$ will approach a Gaussian 


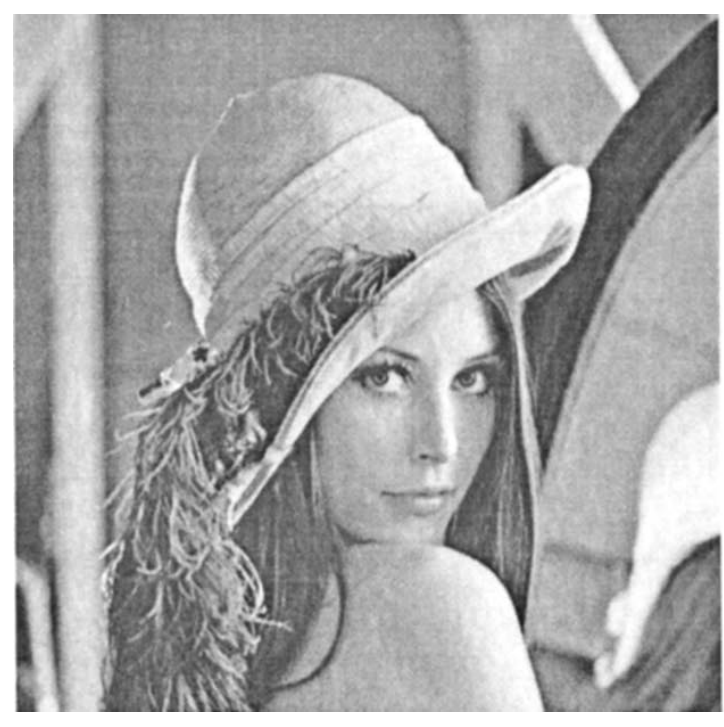

(a)

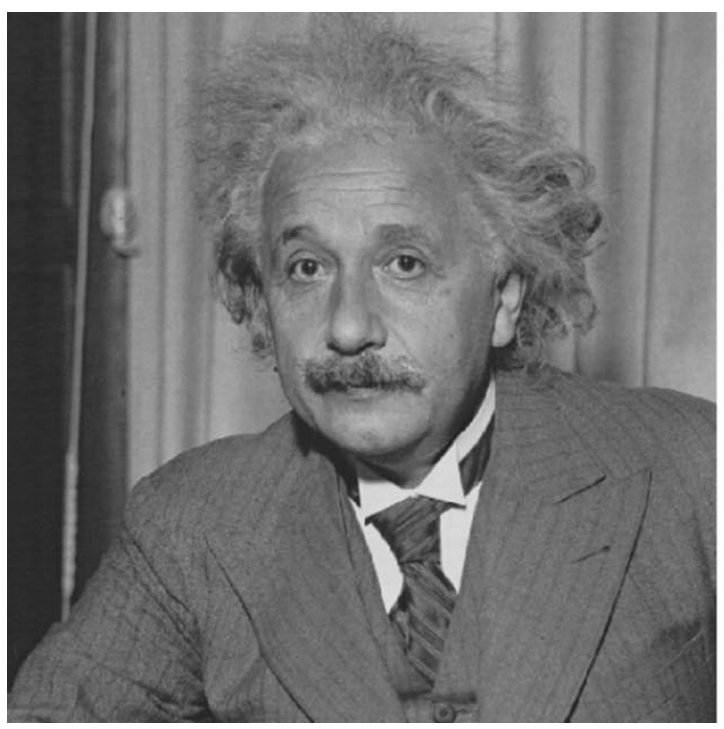

(c)

Fig. 2. (a)-(d) Four of the eight test images.

distribution with mean $M$ and variance $2 M$

$$
p\left(\frac{M \zeta}{\sigma_{0}^{2}}\right)=\frac{1}{2 \sqrt{\pi M}} e^{-(\zeta-M)^{2} / 4 M}
$$

where $p(\cdot)$ denotes the probability density function. Equation (14) implies that

$$
p(\zeta)=\frac{1}{2 \sigma_{0}^{2} \sqrt{\pi / M}} e^{-\left(\zeta-\sigma_{0}^{2}\right)^{2} /\left(2 \sigma_{0}^{4} / M\right)} .
$$

Although Hunt's Gaussian model is simple, it may not be able to model realistic images well. Image signals may be nonstationary in many ways [23] and it is difficult to find the true LSD distributions. However, note that $\zeta$ is obtained by averaging $M+1$ random variables. If $M$ is large enough, the shape of the LSD distribution should not deviate from that in

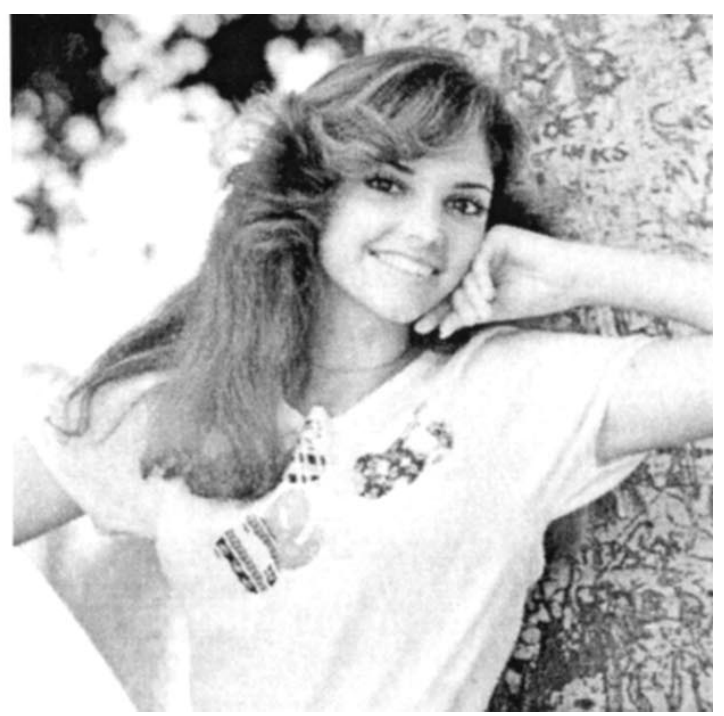

(b)

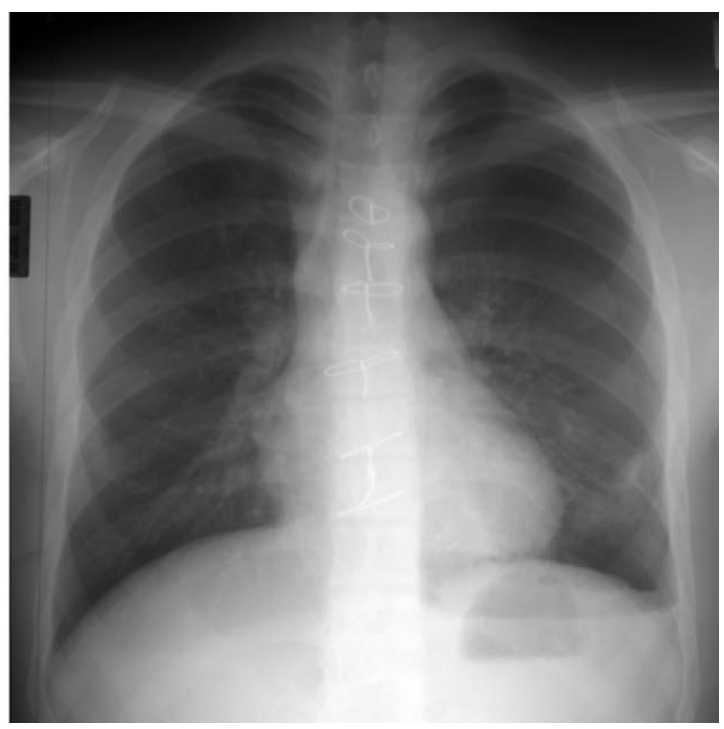

(d)

(15) by much. Thus, one reasonable extension for (15) can be

$$
p(\zeta)=g e^{-|\zeta-\gamma|^{\alpha} / \beta}
$$

where $\alpha, \beta$, and $\gamma$ are distribution parameters and $g$ is a normalized constant. Let $\sigma=\sqrt{\zeta}$, then $\sigma$ is the LSD. From (16), the LSD distribution is obtained as follows:

$$
p(\sigma)=g \sigma e^{-\left|\sigma^{2}-\gamma\right|^{\alpha} / \beta} .
$$

One may ask "how good is the LSD model in (17)?" We have performed some experiments to evaluate this. We chose eight typical images used in general and medical image processing (Fig. 2). We first computed the LSD histograms of these images. Then, for each image, we used the least-squares (LS) method to fit the LSD histograms. The LS method solves 


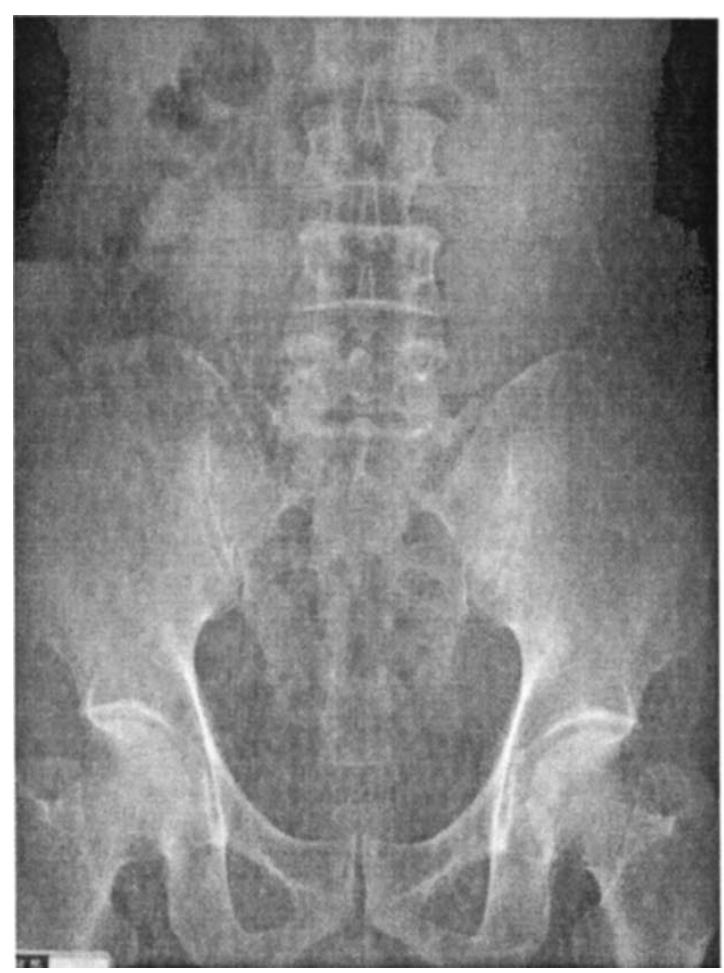

(e)

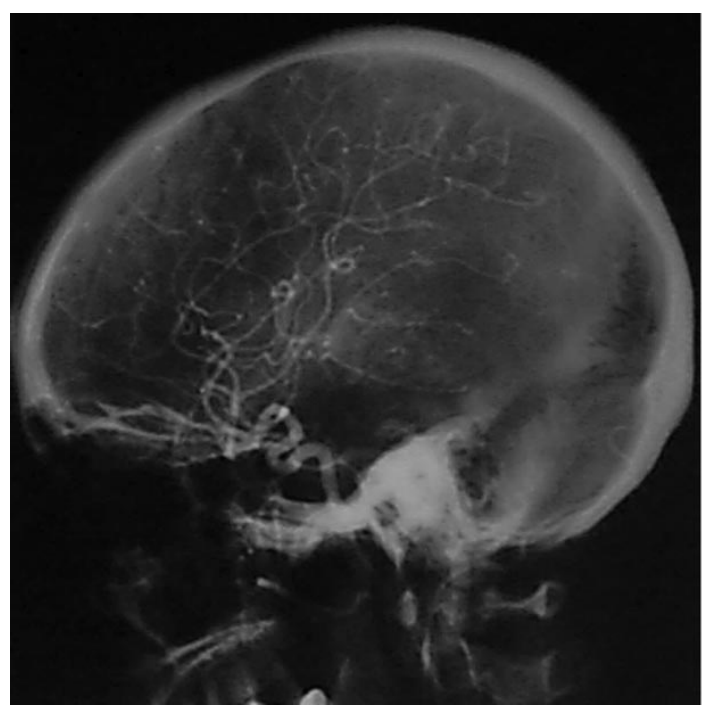

(g)

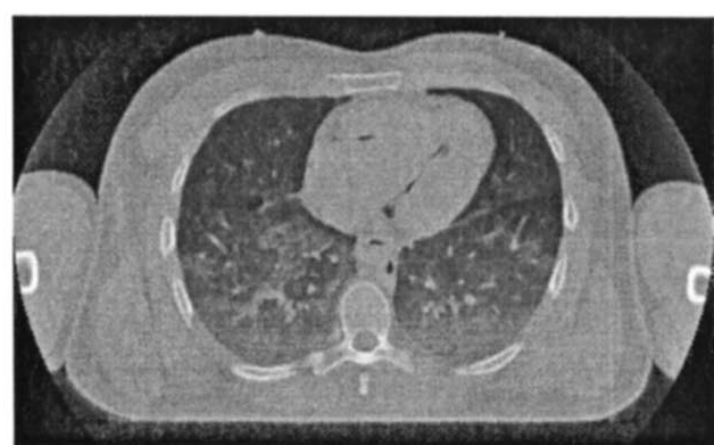

(f)

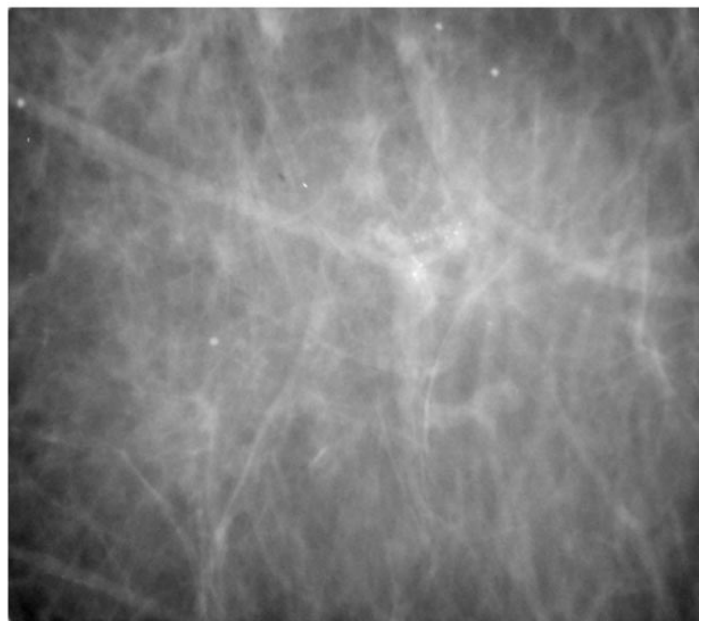

(h)

Fig. 2 (Continued.) (e)-(h) Four of the eight test images.

the following optimization problem

$$
e=\operatorname{Min}_{\alpha, \beta, \gamma} \int[p(\sigma)-h(\sigma)]^{2} d \sigma
$$

where $p(\sigma)$ is the LSD model in (17) and $h(\sigma)$ is the LSD histogram. Assume that the LSD has been quantized into $m+1$ levels. Rewriting the problem in discrete type, we have

$$
e=\operatorname{Min}_{\alpha, \beta, \gamma} \sum_{j=0}^{m}[p(\sigma(j))-h(\sigma(j))]^{2} .
$$

Using (19), we found out the parameters of LSD distributions and they were listed in Table I. The corresponding LSD distributions modeled by (17) and the LSD histograms are shown in Fig. 3. From the Table I, we find that the modeling errors $e$ 's are on the order of $10^{-3}$. It seems that our modeling has sufficiently good results in spite of the image contents. We have also tried different window sizes ranging from $3 \times 3$ to $101 \times 101$ for the test image (d) and found little influence on modeling error. We consider this an important finding since it will be difficult to find a simple model for the grayscale histogram. From empirical results, we finally came to a conclusion that, our LSD model in (17) is applicable to many natural images. 


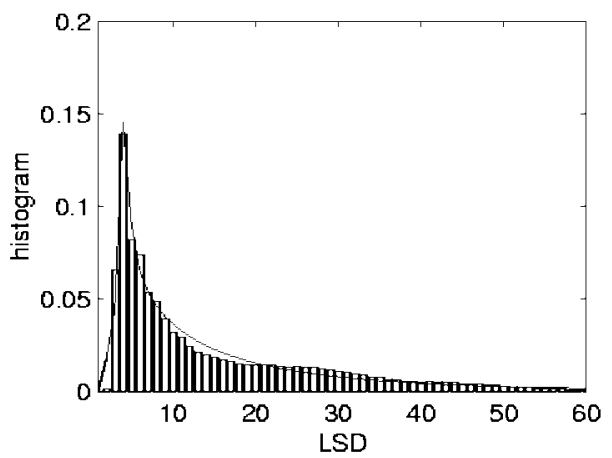

(a)

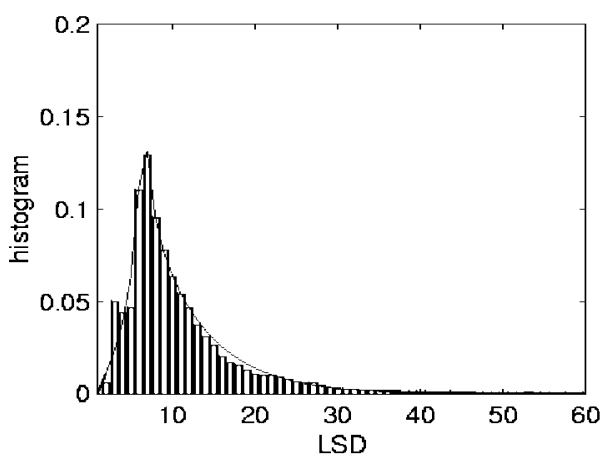

(c)

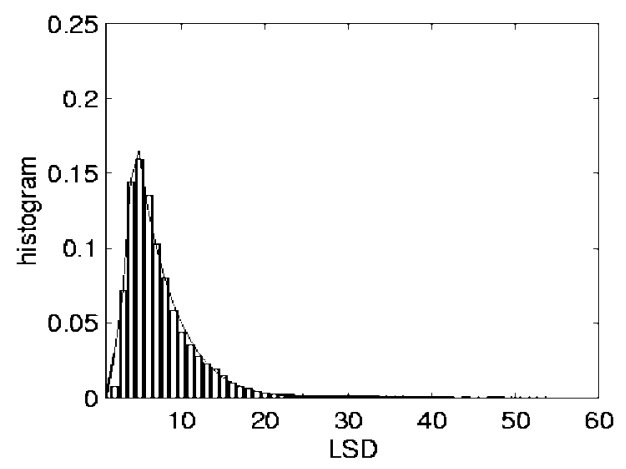

(e)

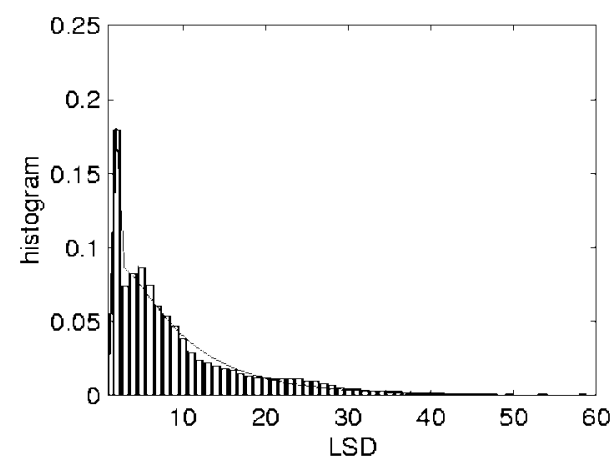

(g)

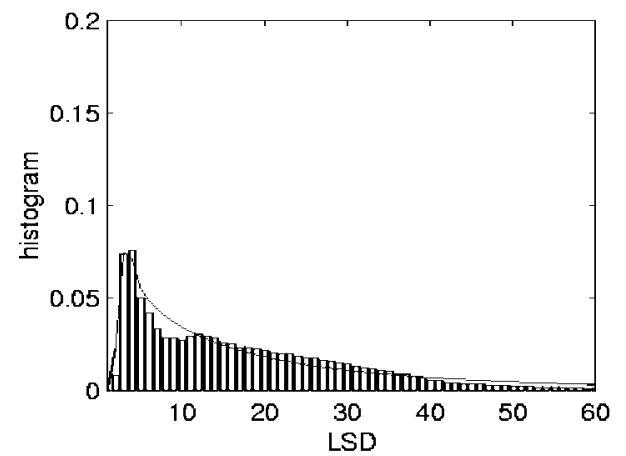

(b)

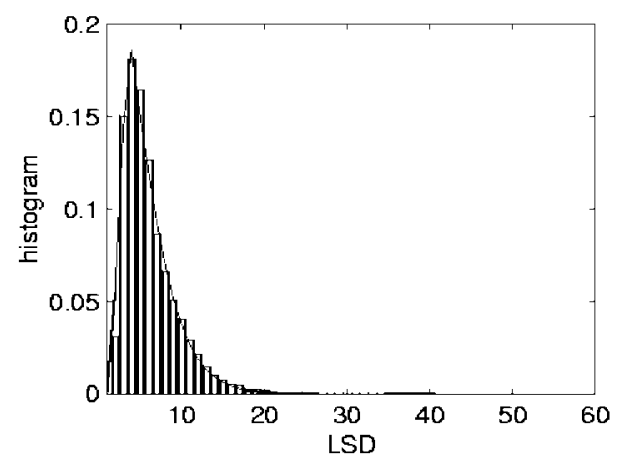

(d)

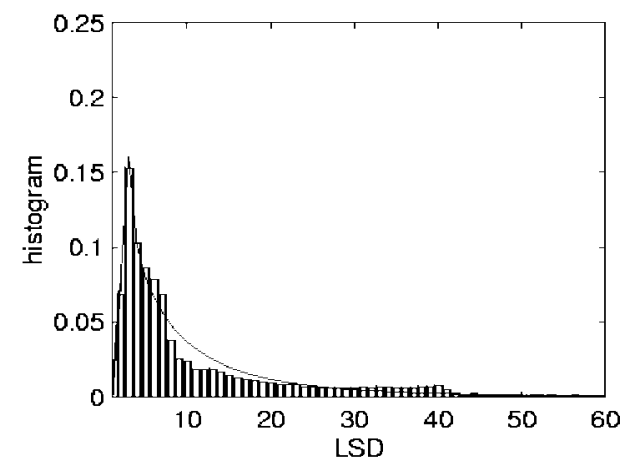

(f)

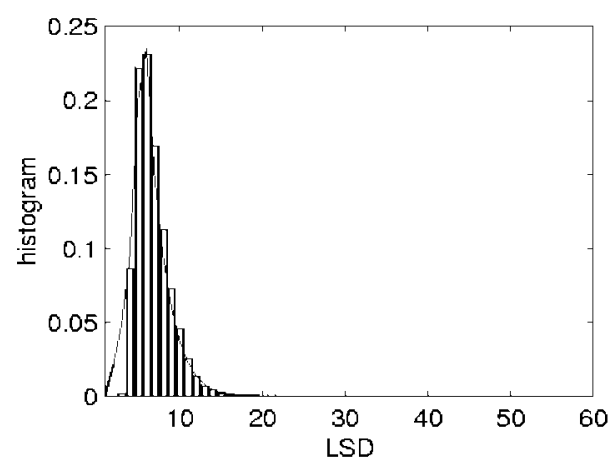

(h)

Fig. 3. The LSD histograms of the eight test images in Fig. 2 and their LS-fitted distributions.

It is relevant to note here that the parameters of the LSD distribution for a given image is strongly dependent on the window size. An experiment for the test image (d) is shown in Fig. 4. As we can see the larger the window size, the wider the LSD distribution will spread. This result can be explained as follows. When we calculate the LSD, we implicitly assume that pixels inside the window are stationary. However, this assumption is not valid for realistic images. In addition, there 
TABLE I

PARAMETERS OF THE LS-FitTED Distributions: Initial Values Are those Inside Parentheses

\begin{tabular}{c||c|c|c|c}
\hline Test inages & Image (a) & Image (b) & Image (c) & Image (d) \\
\hline Image size & $512 \times 512$ & $512 \times 512$ & $512 \times 512$ & $911 \times 911$ \\
\hline Window size & $11 \times 11$ & $11 \times 11$ & $11 \times 11$ & $21 \times 21$ \\
\hline \hline$\alpha$ & $0.1852(0.2)$ & $0.1720(0.5)$ & $0.4336(0.5)$ & $0.5764(0.5)$ \\
\hline$\beta$ & $0.4714(2.0)$ & $0.4653(5.0)$ & $3.0213(2.0)$ & $3.7619(0.5)$ \\
\hline$\gamma$ & $10.1919(10)$ & $5.2858(10)$ & $30.3718(20)$ & $5.7918(5)$ \\
\hline$g$ & 0.4353 & 0.3532 & 0.0441 & 0.1045 \\
\hline$e$ & 0.0012 & 0.0010 & 0.0012 & 0.0009 \\
\hline \hline Test images & Image (e) & Image (f) & Image (g) & Image (h) \\
\hline Image size & $950 \times 700$ & $315 \times 512$ & $390 \times 390$ & $433 \times 488$ \\
\hline Window size & $21 \times 21$ & $11 \times 11$ & $11 \times 11$ & $11 \times 11$ \\
\hline \hline$\alpha$ & $0.5218(0.5)$ & $0.1930(0.2)$ & $0.2884(0.2)$ & $0.6840(0.5)$ \\
\hline$\beta$ & $3.4936(5.0)$ & $1.4203(0.2)$ & $0.9608(0.2)$ & $5.6614(5.0)$ \\
\hline$\gamma$ & $11.5390(10)$ & $2.5856(2)$ & $1.0000(2)$ & $20.0684(5)$ \\
\hline$g$ & 0.0771 & 1.0204 & 0.1800 & 0.0797 \\
\hline$e$ & 0.0007 & 0.0016 & 0.0006 & 0.0042 \\
\hline & & & &
\end{tabular}

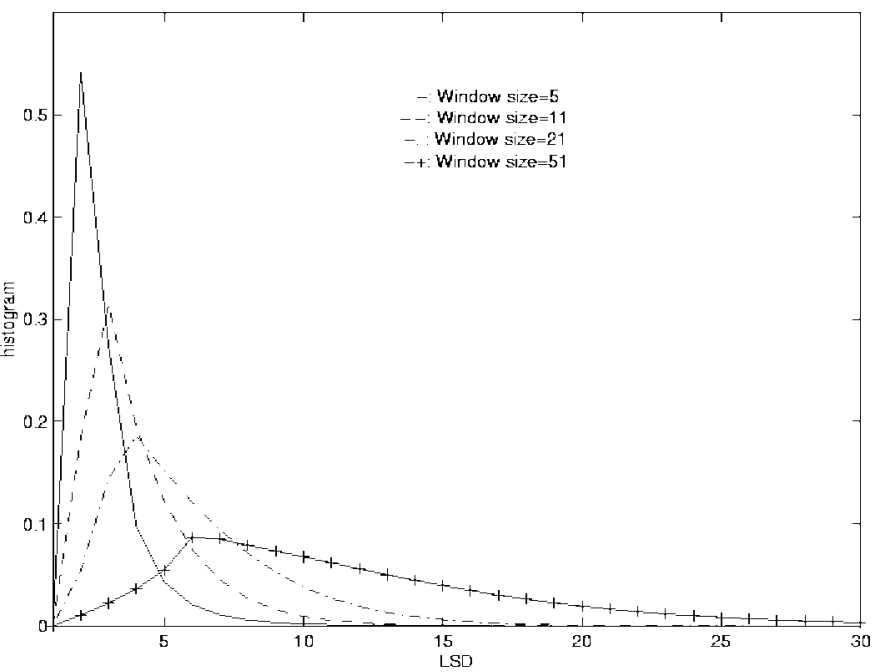

Fig. 4. The LS-fitted LSD distributions of test image Fig. 2(d) in Fig. 2 for varying window sizes.

is a strong correlation between the pixels in a neighborhood. Thus, the computed LSD value will increase as the window size is increased. With this understanding, one may wonder how to determine the window size. There are two steps we can follow. First, we have to define the details. This is important since for different purposes, the definition of details may be different. As we mentioned in Section I, we seek details corresponding to regions with median LSD's. Thus, the second step is to choose a window size that can produce median LSD values for the interested details. Apparently, this choice is dependent on the detail pattern as well as the image resolution. For the same image, if the resolution is increased, the window size should also be increased.

Using our model, a low-contrast image indicates only that the LSD has a small dynamic range. Thus, by transforming the LSD to another variable having a large dynamic range, contrast enhancement is achieved. The problem is how do we perform the transformation. Note that the transformed LSD distribution can have any shape. There are no theoretical results showing that one particular transformation will be better than another.
Although we can perform extensive subjective evaluations to determine the best transformation function, this will be timeconsuming. Here, we propose a simple solution. We use the distribution function described in (17) as the desired LSD distribution. There are at least two reasons for this approach. From experiments, we found that the LSD's of natural images, regardless if the contrast is good or poor, can be modeled well by (17). Thus, if we use (17) as the transformed LSD distribution, the enhanced image may be seen as the result of imaging a natural scene under conditions of good lighting. It is our hope that the enhanced image will not only be good for diagnosis, but also look natural. Our formulation is similar to the conventional histogram transformation. The problem in the conventional histogram transformation is that there is no suitable histogram model to guide the transformation. The histogram of an image can have an arbitrary shape. It will be difficult to construct a simple distribution that can model the histogram adequately. In the following section, we outline a method that performs the proposed transformation easily.

\section{CONTRAST ENHANCEMENT BASED ON THE LSD HISTOGRAM TRANSFORMATION}

In the preceding section, we established a model describing the image LSD distribution, and showed how to use the LS method to find appropriate parameters. Contrast enhancement can be achieved using LSD histogram transformation. In other words, we want the LSD histogram to have a different set of $\alpha$, $\beta$, and $\gamma$ such that the transformed LSD has a larger dynamic range. Contrast gain can then be obtained from the mapping relationship between LSD's before and after transformation.

\section{A. LSD Transformation}

Let $h_{x}\left(\sigma_{x}\right)$ be the image LSD histogram before transformation and $h_{f}\left(\sigma_{f}\right)$ the LSD distribution after transformation. As mentioned previously, $\sigma_{f}=K\left(\sigma_{x}\right)$ and $K(\cdot)$ is the MCG function. From the probability theory, we have

$$
h_{f}\left(\sigma_{f}\right)=\left.h_{x}\left(\sigma_{x}\right)\right|_{\sigma_{x}=K^{-1}\left(\sigma_{f}\right)} \cdot\left|\frac{d \sigma_{x}}{d \sigma_{f}}\right| .
$$

Since $K(\cdot)$ is a monotonic function, and $\sigma_{x}$ and $\sigma_{f}$ are nonnegative, $d \sigma_{x} / d \sigma_{f}$ is a positive value. The absolute value sign in (20) can be ignored. Integrating both sides, the equation can be represented as

$$
\int h_{f}\left(\sigma_{f}\right) d \sigma_{f}=\int h_{x}\left(\sigma_{x}\right) d \sigma_{x}
$$

Assume that the desired parameters in (17) have been determined, say, $\alpha_{f}, \beta_{f}$, and $\gamma_{f}$. Now, approximating the LSD histogram for the output image as the model in (17), we have

$$
h_{f}\left(\sigma_{f}\right)=g_{f} \sigma_{f} e^{-\left|\sigma_{f}^{2}-\gamma_{f}\right|^{\alpha_{f}} / \beta_{f}} .
$$

Let the LSD be quantized into $m+1$ levels and denote the quantized LSD before and after transformation as $\sigma_{x}(k)$ and $\sigma_{f}(k), k=0,1, \cdots, m$, respectively. Rewriting (21) in its 


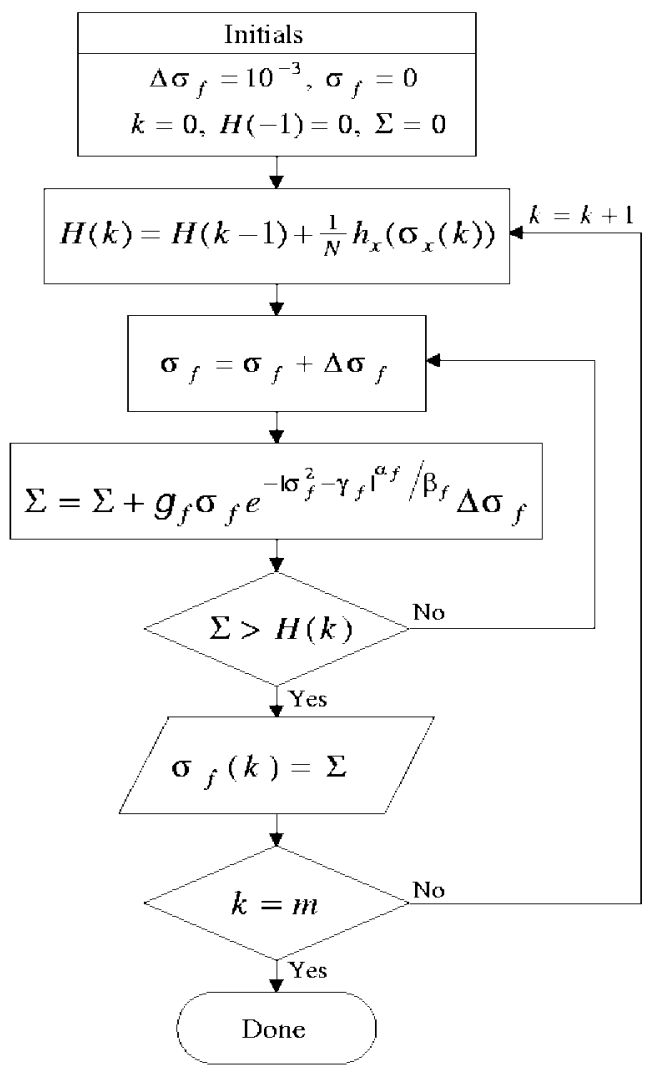

Fig. 5. The flowchart for computing the LSD transformation.

discrete form, we have

$$
\begin{aligned}
& \int_{\sigma_{f}(0)}^{\sigma_{f}(k)} g_{f} \sigma_{f} e^{-\left|\sigma_{f}^{2}-\gamma_{f}\right|^{\alpha_{f} / \beta_{f}}} d \sigma_{f}=\frac{1}{N} \sum_{j=0}^{k} h_{x}\left(\sigma_{x}(j)\right) \\
& \quad k=0,1, \cdots, m
\end{aligned}
$$

where $N$ is the total number of pixels in the image. We assume $\sigma_{f}(0)=0$. The transformation function $K(\cdot)$, which represents the transformation from $\sigma_{x}(k)$ to $\sigma_{f}(k)$, can then be obtained by solving (23). Note that the unknown in (23) is $\sigma_{f}(k), k=0,1, \cdots, m$. To obtain accurate solutions for $\sigma_{f}(k)$ is complicated and not necessary. Here, we use a simple method depicted by the flowchart in Fig. 5. Here, the increment $\triangle \sigma_{f}$ was set at $10^{-3}$. We can obtain more accurate results by using a smaller $\Delta \sigma_{f}$ value at the cost of higher computing time.

We can also use the other transformation method, which replaces the empirical histogram with the LS-fitted distribution. Let the LS-fitted parameters in (17) be $\alpha_{x}, \beta_{x}$, and $\gamma_{x}$. Equation (23) then becomes

$$
\begin{aligned}
& \int_{\sigma_{f}(0)}^{\sigma_{f}(k)} g_{f} \sigma_{f} e^{-\left|\sigma_{f}^{2}-\gamma_{f}\right|^{\alpha_{f} / \beta_{f}}} d \sigma_{f} \\
& \quad=\int_{\sigma_{x}(0)}^{\sigma_{x}(k)} g_{x} \sigma_{x} e^{-\left|\sigma_{x}^{2}-\gamma_{x}\right|^{\alpha_{x}} / \beta_{x}} d \sigma_{x}, \quad k=0,1, \cdots, m .
\end{aligned}
$$

If the $\operatorname{LSD} \sigma_{x}(i, j)$ is at the $(k+1)$ th level, i.e., $\sigma_{x}(k)$, the MCG in (8) is then $\sigma_{f}(k)$. This completes the derivation of our ACE algorithm.

\section{B. Determination of Parameters}

The final problem is how to choose appropriate parameters for (17) in order to perform the LSD transformation. Since there are three parameters $\alpha, \beta$, and $\gamma$, direct trial-and-error requires a lot of work. Here, we developed a simple method to overcome this problem. Our idea is to modify the gain function in the CGT algorithm using the proposed LSD model. Consider the CGT algorithm described by $\sigma_{f}=G \sigma_{x}$ where $G$ is a constant. Then

$$
\begin{aligned}
p\left(\sigma_{f}\right) & =\left.p\left(\sigma_{x}\right)\right|_{\sigma_{x}=G^{-1} \sigma_{f}} \cdot\left|\frac{d \sigma_{x}}{d \sigma_{f}}\right| \\
& =g_{x} G^{-1} \sigma_{f} e^{-\left|G^{-2} \sigma_{f}^{2}-\gamma_{x}\right|^{\alpha_{x}} / \beta_{x}} \cdot G^{-1} \\
& =g_{f} \sigma_{f} e^{-\left|\sigma_{f}^{2}-\gamma_{f}\right|^{\alpha_{x}} / \beta_{f}}
\end{aligned}
$$

where $g_{f}=g_{x} G^{-2}$ and

$$
\beta_{f}=G^{2 \alpha_{x}} \beta_{x}, \quad \gamma_{f}=G^{2} \gamma_{x} .
$$

Thus, the CGT algorithm simultaneously increases $\gamma$ and $\beta$. As mentioned previously, the CGT algorithm suffers the ringing effect. To eliminate this problem, CG in the high LSD region (marked $\mathbf{A}$ in Fig. 1) must be reduced. This can be achieved by increasing $\alpha$. It can be seen that $\alpha$ controls the characteristics of the distribution tail. The larger the $\alpha$, the shorter the distribution tail and the smaller the CG in the high LSD region. Parameter $\gamma$ has a large influence on the mode position of the LSD distribution. If $\gamma$ is increased, the mode position moves farther from the origin. Then, the MCG will have a larger value in the low LSD regions. Note that increasing $\alpha$ will magnify the MCG in the low LSD region and this is not desirable. Thus, $\gamma$ should be decreased to compensate for this effect.

Using the procedure outlined above, we can adjust the three parameters to obtain the desired transformation. However, it is found that directly adjusting $\alpha, \beta$, and $\gamma$ is not efficient. Using the result in (26), we now propose another way to do the job. Let

$$
\alpha_{f}=A \alpha_{x}, \quad \beta_{f}=E^{2 \alpha_{f}} \beta_{x}, \quad \gamma_{f}=F^{2} \gamma_{x} .
$$

Instead of $\alpha, \beta$, and $\gamma$, we use $A, E$, and $F$ to obtain the desired transformation. Experiments show that this approach greatly facilitates the parameter determination. From the discussion in the last paragraph, it is obvious that $A>1$, and $E>F$. We now summarize the procedure for parameter determination as follows.

1) Choose a proper constant $E$.

2) Choose $A$ such that the MCG can suppress the ringing artifacts in the high LSD region.

3) Choose $F$ such that the high contrast region can be moved to the desired place.

To obtain the best results, this procedure can be carried out repeatedly. From our experience, we found that typical values 


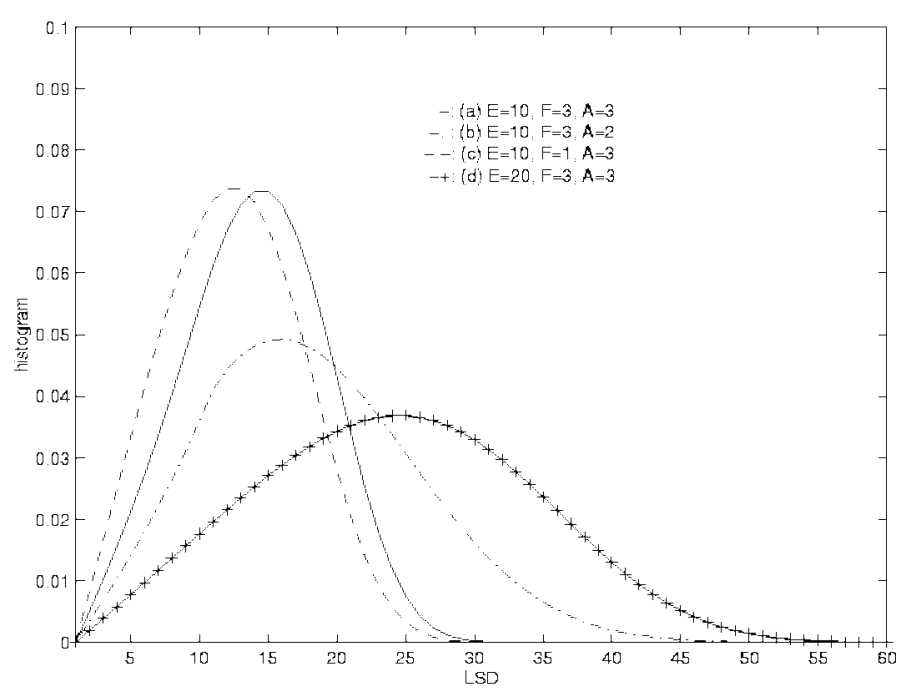

Fig. 6. The modeled LSD distributions for varying parameters $A, E$, and $F$.

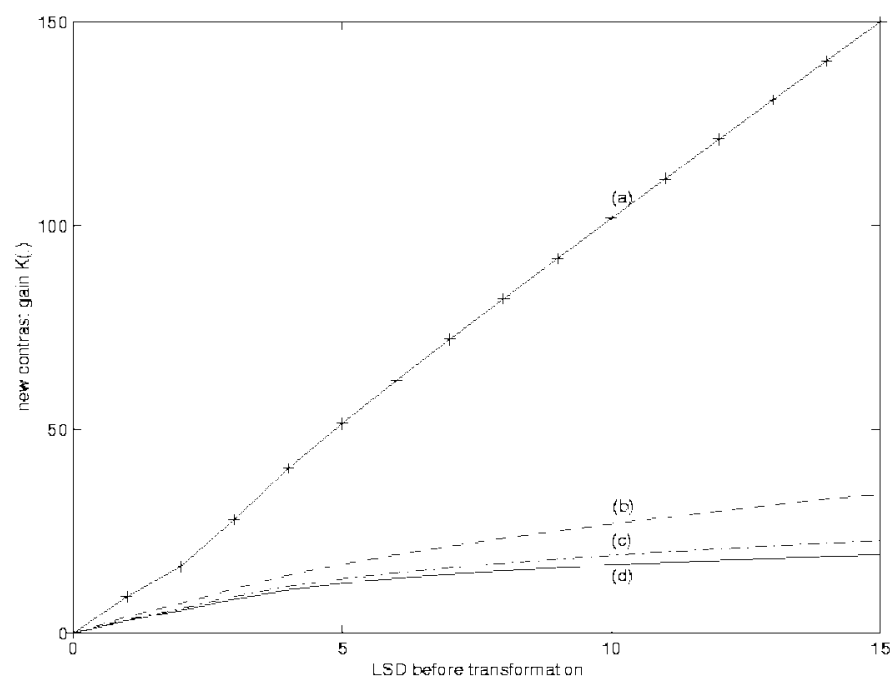

Fig. 7. The characteristics of parameter $\alpha[A=1,2,3,4$ for (a)-(d)].

for the parameters can be $A=2 \sim 5, E=10 \sim 30$, and $F=2 \sim 4$.

We next provide examples to show the influences of parameters $A, E$, and $F$ on the MCG function. This will be helpful for understanding the proposed procedure. Let $\alpha_{x}=0.6$, $\beta_{x}=5.5$, and $\gamma_{x}=(3.4)^{2}$. In Fig. 7, we plot the MCG's for varying $A$ ( $E=10$ and $F=3$ ). As we expect, the larger the $A$, the smaller the MCG in the high LSD region. We also plot the transformed LSD distributions in Fig. 6. From the distributions labeled by (a) and (b) in the figure, we can see that $A$ does control the tail characteristics. The MCG function for varying $F$ is shown in Fig. $8(A=1$ and $E=10)$. The transformed LSD distributions for two different $F$ 's are shown in Fig. 6, in which they are labeled as (a) and (c). The LSD distribution shapes look alike, but the mode positions are clearly different. In Fig. 9, we plot the MCG function for varying $E(A=1$ and $F=1)$. As we can see, the role of $E$ is very similar to the constant gain in the CGT algorithm. The MCG functions are almost linear and the larger the $E$, the

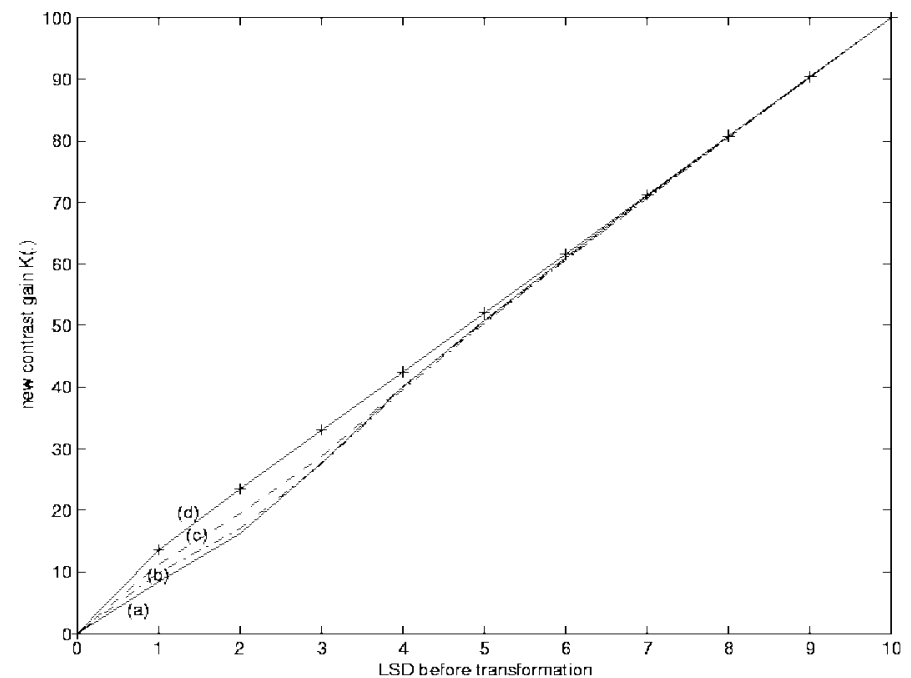

Fig. 8. The characteristics of parameter $\gamma[F=1,5,7,10$ for (a)-(d)].

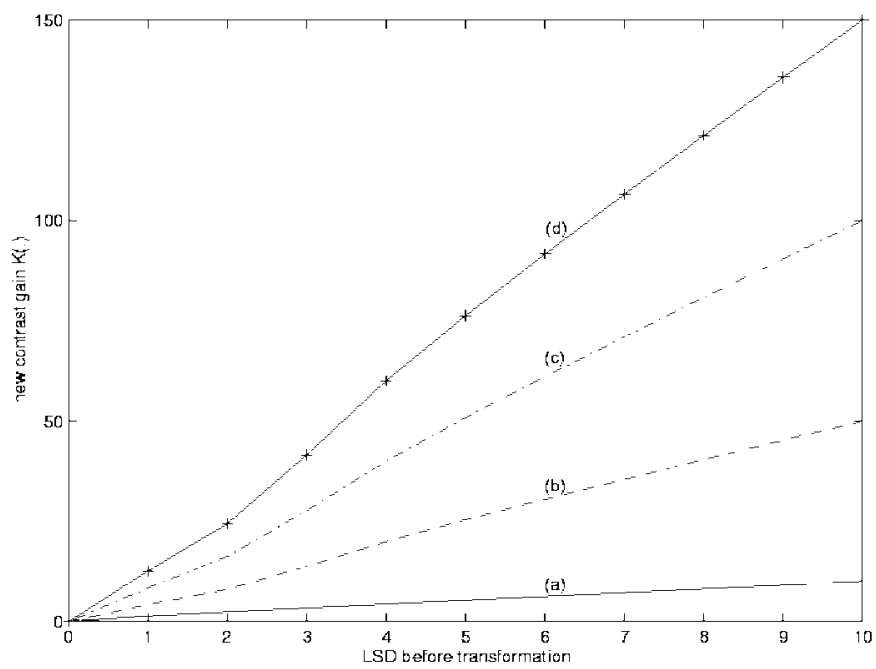

Fig. 9. The characteristics of parameter $\beta[E=1,5,10,15$ for (a)-(d)].

steeper the MCG function. This is not surprising since $A$ is small in this case. The transformed LSD distributions for two different $E$ 's are shown in Fig. 6, in which they are labeled (a) and (d). We find that the one with the larger $E$ looks like a linear stretch of the one with the smaller $E$.

Once the parameters are determined, we can perform the LSD transformation to enhance the image. We can now summarize the whole procedure for implementing our scheme.

1) Calculate the LSD histogram of the image and use the LS method to find the corresponding parameters for (17).

2) Find the desired parameters $A, E$, and $F$ using the procedure outlined above.

3) Perform the LSD transformation in (23) using (27) and find the mapping function.

4) Use the mapping function to carry out the enhancement described in (8). 


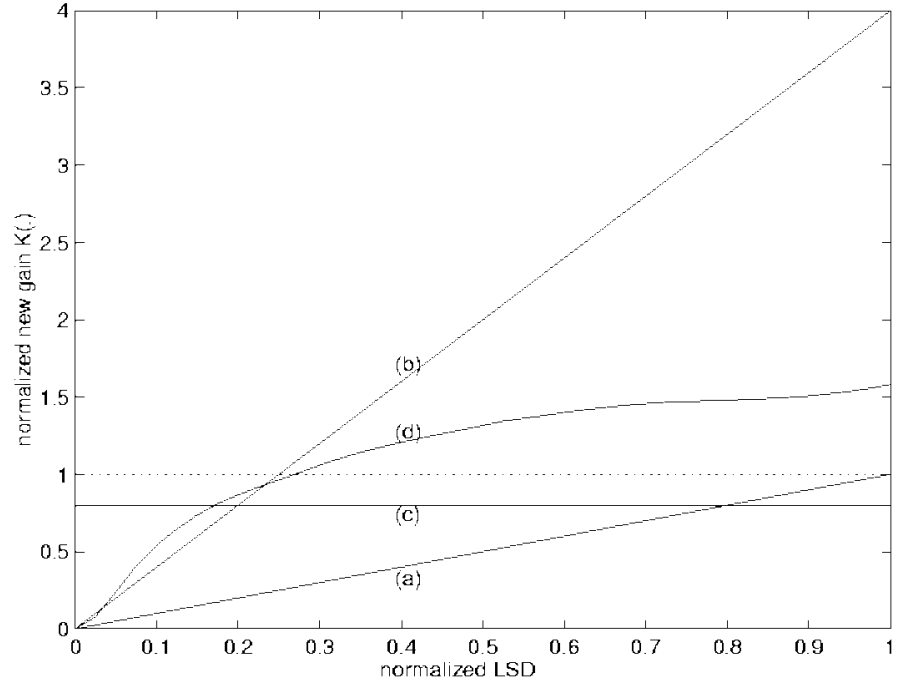

Fig. 10. The CG $K(i, j)$ : (a) the CGT algorithm $(C=1)$, (b) the CGT algorithm $(C=4)$, (c) the IPLSD algorithm $(D=0.8)$, and (d) the proposed algorithm.

Our method is based on the LSD histogram transformation. In other words, images with the same LSD histogram may share the same mapping functions. If the window size and image resolution remain the same, we can expect that LSD histograms will be similar for the same types of images taken from the same imaging system. Thus, we require only one common nonlinear LSD mapping function. To reduce computations, the MCG function can be tabulated in advance of processing. Then, the computational complexity of the proposed algorithm is identical to the conventional IPLSD algorithm. Note that both algorithms have to compute local means as well as LSD's. In contrast, the CGT algorithm needs only to compute the local means. Thus, the CGT algorithm requires fewer computations. To further reduce the computational complexity of the proposed algorithm, some fast algorithms for local mean and LSD computations [17] can be applied.

\section{Simulations}

The chest X-ray image in Fig. 2(d) was used to perform simulations. The image size was $911 \times 911$ and the gray-scale consisted of 256 levels. The window size for the local mean and LSD calculation was chosen as $21 \times 21$. The details to be enhanced were lung lesions and indistinct ribbed patterns. Here we assumed that global contrast was acceptable and focused only on the enhancement of the local contrast. The MCG $K(i, j)$ used for the simulations are shown in Fig. 10. Since the cumulative value of the LSD histogram was almost one when the LSD $=30$ [as shown in Fig. 3(d)], the LSD was normalized with respect to the value of 30. Pixels with LSD's beyond 30 had their CG's simply clipped. The four MCG's in Fig. 10 correspond to the following algorithms.

a) The CGT algorithm in (4) with $C=1$.

b) The CGT algorithm in (4) with $C=4$.

c) The IPLSD algorithm in (5) with $D=0.8$.

d) The proposed algorithm with parameters of $A=4$, $E=25$, and $F=3$.

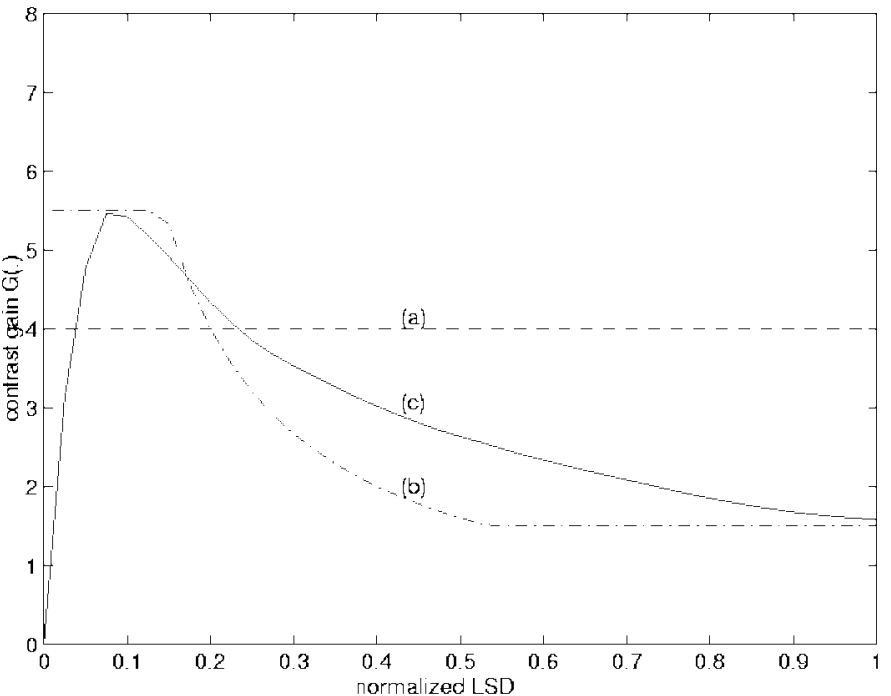

Fig. 11. The CG $G(i, j)$ : (a) the CGT algorithm $(C=4)$, (b) the clipped IPLSD algorithm, and (c) the proposed algorithm.

To avoid large noise amplification, the IPLSD gain was clipped at a maximum $G(i, j)$ of 5.5 and a minimum $G(i, j)$ of 1.5. The corresponding CCG functions of the test algorithms are also depicted in Fig. 11. It is apparent that the proposed algorithm has high $G(i, j)$ in the detail region (median LSD's) and has small $G(i, j)$ in the smooth and edge regions (small and large LSD's).

The enhanced images are shown in Fig. 12. A rectangle with gray value of 50 was inserted into the original image to evaluate the ringing effect of the test algorithms. From Fig. 12, we find that the CGT algorithm has a severe ringing artifact. This is due to the high constant CG in the edge region. The ringing effect does not show up in the IPLSD and the proposed algorithm. Note that the IPLSD algorithm renders heavy enhancement of normal vascular details. The overemphasized vascular details in the lungs may look like calcification and affect the diagnosis of lung lesions [16]. In our algorithm, the vascular structure is properly handled and the enhanced result does not cause a disturbance.

A simple quantitative analysis was used to evaluate enhancement quality [25], [26]. The image was first segmented into three regions: smooth, detail, and edge regions. The average local variances (ALV's) in the three regions were then calculated as quality measures: the ALV in the smooth region (ALVS), the ALV in the detail region (ALVD), and the ALV in the edge region (ALVE). The ALV in a certain region is defined as follows:

$$
\mathrm{ALV}=\frac{1}{N_{r}} \sum_{(i, j) \in R} \sigma_{x}^{2}(i, j)
$$

where $R$ is the region, $N_{r}$ is the number of pixels in $R$, and $\sigma_{x}^{2}(i, j)$ is the local variance defined in (2). It is apparent that a good enhancement algorithm should give reasonably high ALVD and low ALVS and ALVE. In our analysis, image segmentation was performed by LSD thresholding; each pixel 


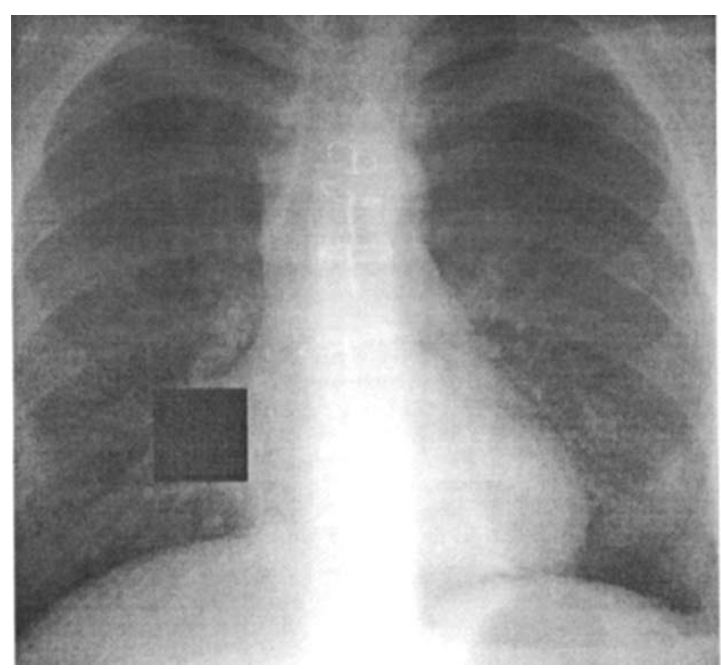

(a)

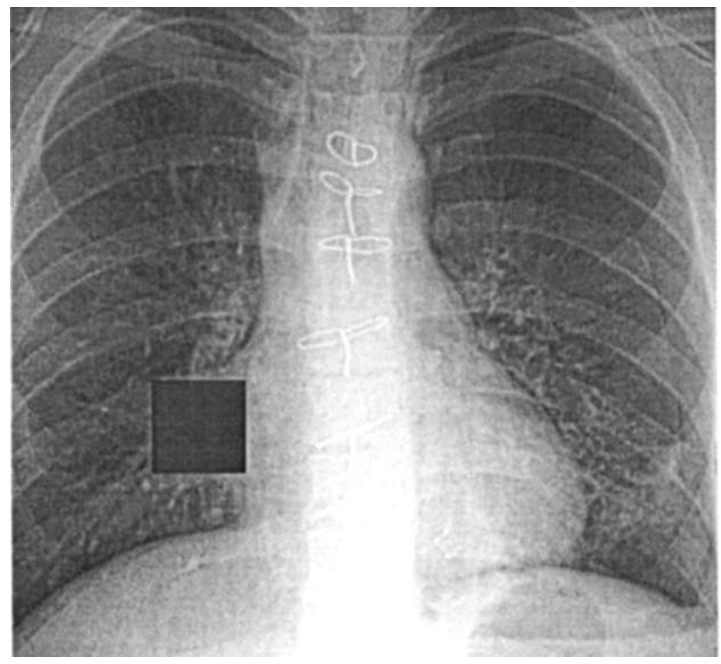

(c)

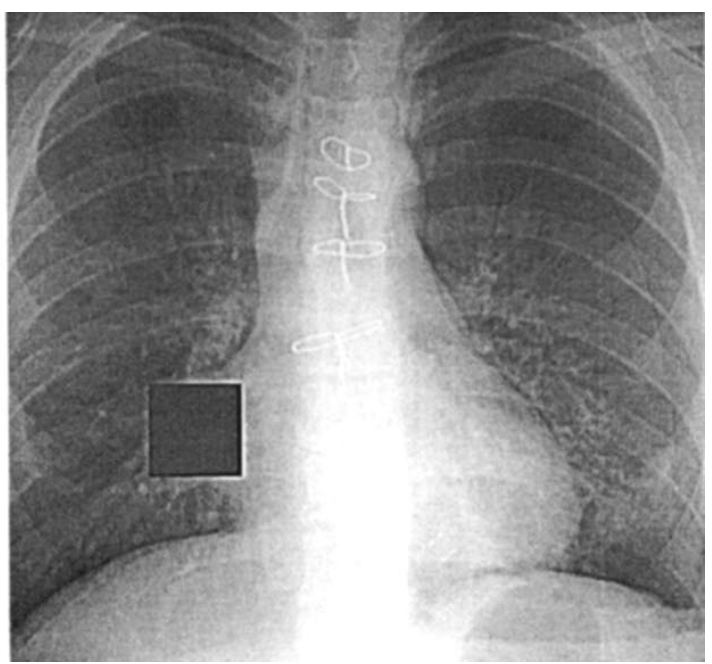

(b)

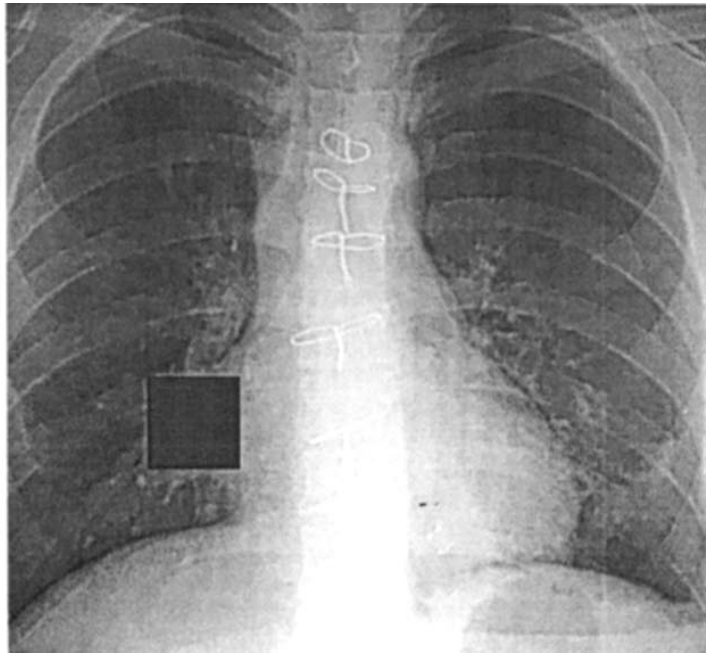

(d)

Fig. 12. Results of contrast enhancement for test image Fig. 2(d): (a) original, (b) the CGT algorithm, (c) the IPLSD algorithm, and (d) the proposed algorithm.

was classified according to the following rules:

$$
\begin{aligned}
\text { LSD }<T_{1} & \rightarrow \text { Smooth Region } \\
T_{1} \leq \mathrm{LSD}<T_{2} & \rightarrow \text { Detail Region } \\
T_{2} \leq \mathrm{LSD} & \rightarrow \text { Edge Region }
\end{aligned}
$$

where $T_{1}$ and $T_{2}$ are two parameters that can be chosen to meet different requirements. In our case, we let $T_{1}=3$ and $T_{2}=12$. Using this simple segmentation scheme, we found that pixels of $28.12 \%$ are in the smooth region, $66.67 \%$ in the detail region, and $5.21 \%$ in the edge region. These regions are shown in Fig. 13. Since we assumed that the vascular structure is not critical, the smooth region was chosen to include some vascular details. The three measures were calculated and listed in Table II. It is seen that the ALVE for the CGT algorithm is significantly higher than others. This indicates severe ringing artifacts. The ALVD for the IPLSD algorithm is close to that for the proposed algorithms, however, the ALVS for the IPLSD algorithm is much larger than that for the proposed algorithm. This explains why the vascular structure is overenhanced in

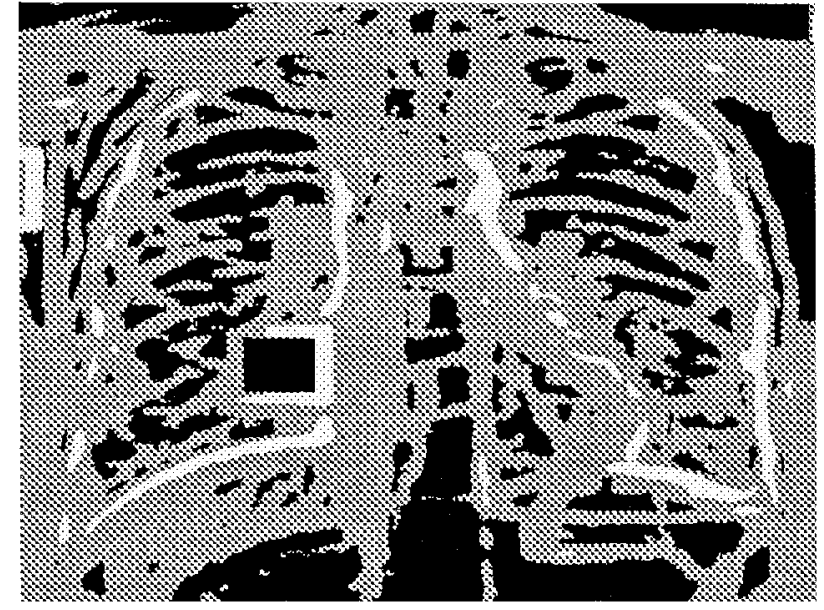

Fig. 13. Segmentation of test image (d) in Fig. 2; smooth (dark) region, detail (gray) region, and edge (white) region.

the IPLSD algorithm. Note that the performance of these three measures strongly depend on the segmentation scheme and parameter $\left(T_{1}, T_{2}\right)$ setting. Although our segmentation scheme 


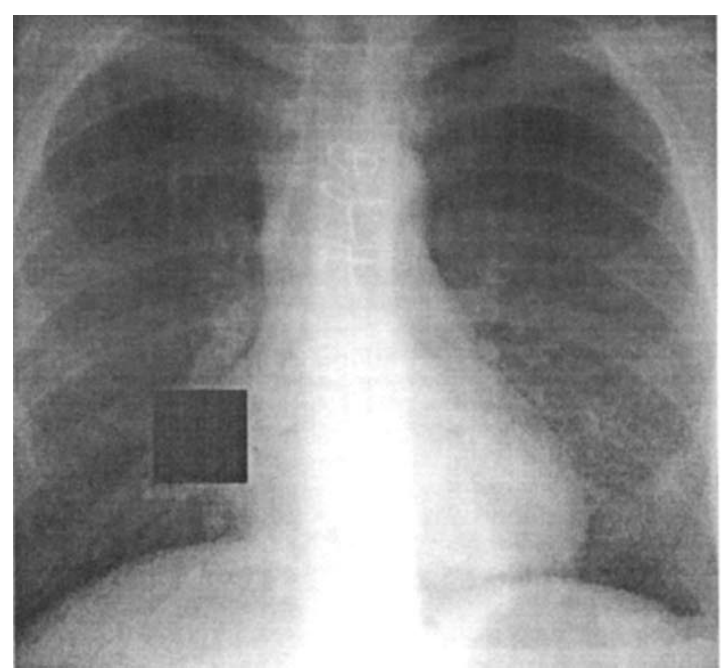

(a)

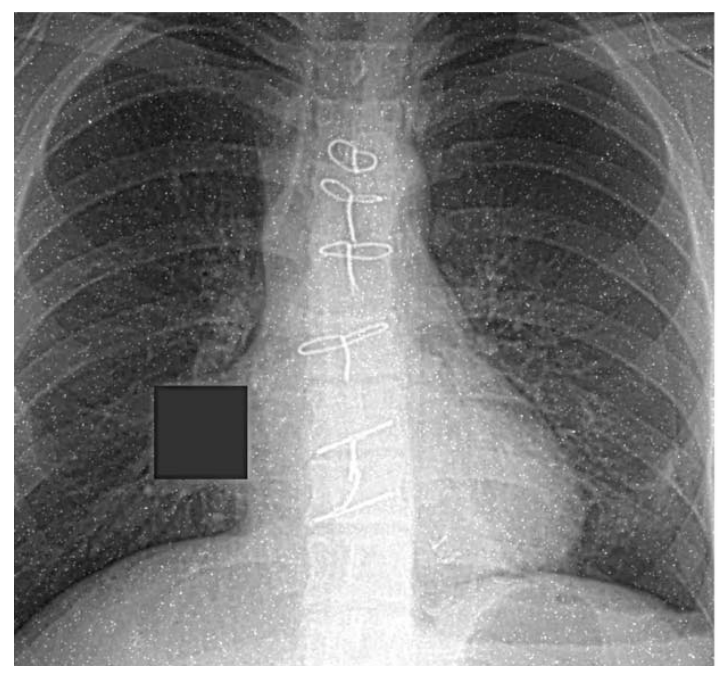

(c)

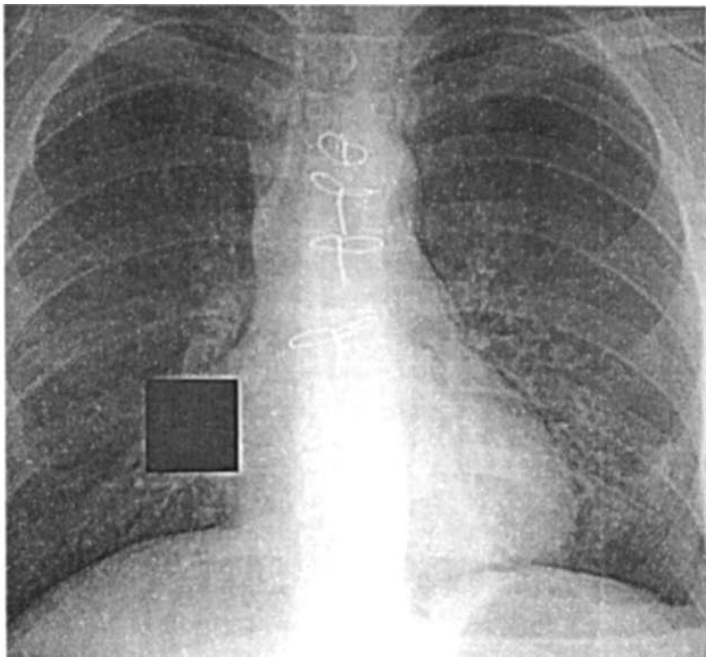

(b)

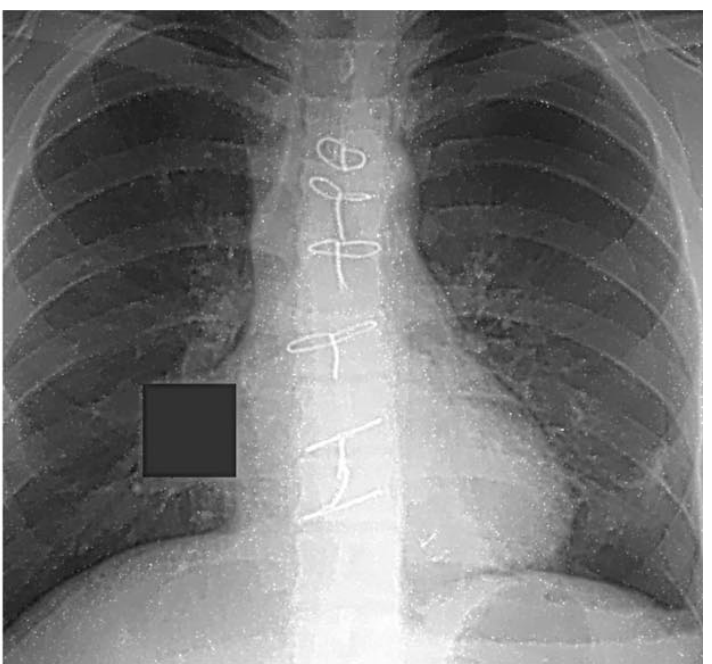

(d)

Fig. 14. Results of contrast enhancement for test image (d) in Fig. 2: (a) original image with $2 \%$ uniform noise, (b) the CGT algorithm, (c) the IPLSD algorithm, and (d) the proposed algorithm.

TABLE II

Comparison of Three AlV Measures For the Test Algorithms

\begin{tabular}{r||r|r|r|r}
\hline Measures & Original Image & CG Algorithm & IPLSD Algorithm & Proposed Algorithm \\
\hline \hline ALVS & 5.0776 & 29.0891 & 48.3439 & 19.2759 \\
\hline ALVD & 38.5072 & $\mathbf{9 8 . 1 4 0 3}$ & 135.9114 & 127.0441 \\
\hline ALVE & 316.6101 & 920.2396 & 506.6209 & 508.8403 \\
\hline
\end{tabular}

is simple, these measures still provide useful information for evaluation of the contrast enhancement.

To further test the noise enhancement property, we randomly added a $2 \%$ uniform noise with values between zero and six to the image. Note that the early calcification lesions usually cause white spots in X-ray images. To show the averse effect of noise overenhancement, we only considered noise with positive values. For better visibility, each noise sample covered four contiguous pixels. The noisy and enhanced images are shown in Fig. 14. From this figure, we find that noise is almost invisible before enhancement. However, it becomes apparent after enhancement. It is clear that the IPLSD algorithm is more likely to enhance noise. The proposed algorithm does not enhance noise in the small and high LSD regions. From these results, we can conclude that the nonlinear CG is more effective and can properly handle the ringing artifacts and noise overenhancement problem.

We have shown that the proposed algorithm is useful in processing chest X-ray images. To test the effectiveness of the proposed algorithm on other types of images, we 


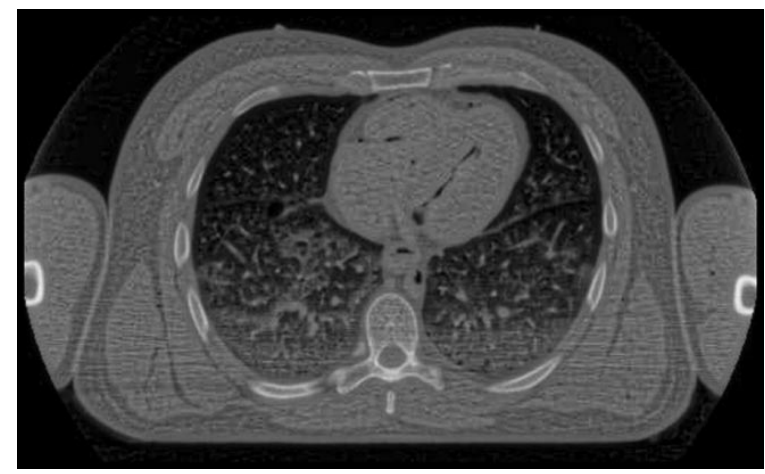

(a)

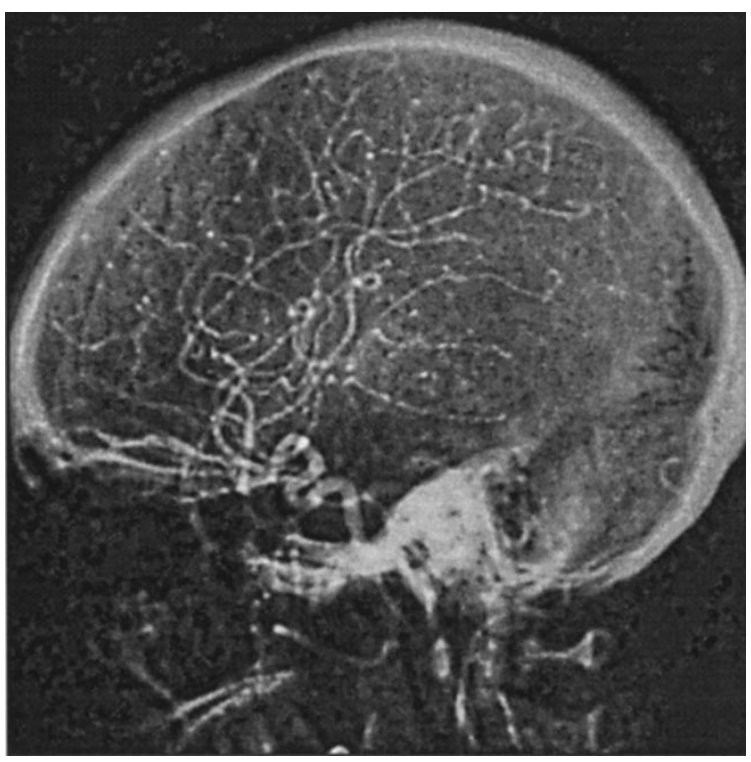

(b)

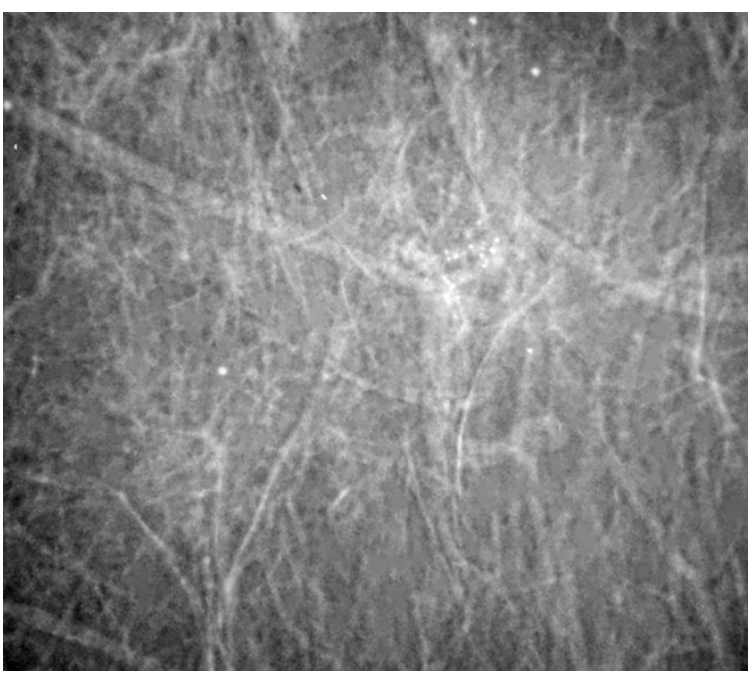

(c)

Fig. 15. Results of contrast enhancement for test images Fig. 2(f)-(h): (a) thorax CT, (b) cerebral angiography, and (c) mammography.

also conducted simulations using the native thorax computed tomography (CT), cerebral angiography, and mammography images shown in Fig. 2(f)-(h). The parameters $(A, E, F)$ used for the enhancement were $(5,25,3),(5,25,3)$, and $(3.5,16$,
3), respectively. The enhanced images are shown in Fig. 15. From these images, we found that the details were efficiently enhanced.

\section{CONCLUSIONS}

The ACE algorithm is a well-known technique for medical image processing. Conventional approaches suffer from noise overenhancement and ringing artifacts. In this paper, we presented a new ACE algorithm to overcome these problems. We first proposed a mathematical model for the LSD distribution. Based on this model, we formulated ACE as an LSD histogram transformation problem. There are three parameters in our model which could be chosen to meet different requirements. Finally, detailed simulations were carried out using a chest $\mathrm{X}$-ray image, showing that our method adequately enhances details and produces little noise overenhancement and few ringing artifacts. Other types of medical images are also tried and effectively enhanced results were also observed. Note that we can separate an image into several regions and use different gain functions for different regions. This may produce even better results. Research in this area is continuing.

\section{ACKNOWLEDGMENT}

The authors would like to thank M. Richardson, M.D. in the Radiology Department, University of Washington, Seattle, WA, and Y.-C. Lee in the Department of Medicine, National Taiwan University, Taipei, Taiwan, for their helpful support in providing medical images and valuable suggestions.

\section{REFERENCES}

[1] A. Laine, J. Fan, and W. Yang, "Wavelets for contrast enhancement of digital mammography," IEEE Eng. Med. Biol. Mag., vol. 14, no. 6, pp. 536-550, 1995.

[2] W. Qian, L. P. Clarke, B. Zheng, M. Kallergi, and R. Clark, "Computer assisted diagnosis for digital mammography," IEEE Eng. Med. Biol. Mag., vol. 14, no. 6, pp. 561-568, 1995.

[3] A. N. Netravali and B. G. Haskeli, Digital Pictures: Representation and Compression. New York: Plenum, 1988, ch. 4.

[4] R. C. Gonzalez and R. E. Woods, Digital Image Processing. New York: Addison-Wesley, 1992.

[5] M. A. Sid-Ahmed, Image Processing: Theory, Algorithms, and Architectures. New York: McGraw-Hill, 1995, ch. 4.

[6] J. D. Fahnestock and R. A. Schowengerdt, "Spatially variant contrast enhancement using local range modification," Opt. Eng., vol. 22, no. 3, pp. 378-381, 1983.

[7] I. Altas, J. Louis, and J. Belward, "A variational approach to the radiometric enhancement of digital imagery," IEEE Trans. Image Processing, vol. 4, pp. 845-849, June 1995.

[8] R. H. Sherrir and G. A. Johnson, "Regionally adaptive histogram equalization of the chest," IEEE Trans. Med. Imag., vol. MI-6, pp. 1-7, Jan. 1987.

[9] S. M. Pizer, J. B. Zimmerman, and E. V. Staab, "Adaptive grey level assignment in CT scan display," J. Comput. Assist. Tomogr., vol. 8, no. 2, pp. 300-305, 1984.

[10] S. M. Pizer, E. P. Amburn, J. D. Austin, R. Cromartie, A. Geselowitz, T Greer, B. H. Romeny, J. B. Zimmerman, and K. Zuiderveld, "Adaptive histogram equalization and its variations," Comput. Vision, Graphics, Image Processing, vol. 39, pp. 355-368, 1987.

[11] J. B. Zimmerman, S. M. Pizer, E. V. Staab, J. R. Perry, W. McCartney, and B. C. Brenton, "An evaluation of the effectiveness of adaptive histogram equalization for contrast enhancement," IEEE Trans. Med. Imag., vol. 7, pp. 304-312, Aug. 1988.

[12] J. S. Lee, "Digital image enhancement and noise filtering by using local statistics," IEEE Trans. Pattern Anal. Machine Intell., vol. PAMI-2, pp. 165-168, Feb. 1980. 
[13] G. Deng, L. W. Cahill, and G. R. Tobin, "The study of logarithmic image processing model and its application to image enhancement," IEEE Trans. Image Processing, vol. 4, pp. 506-512, Apr. 1995.

[14] H. P. McAdams, G. A. Johnson, S. A. Suddarth, R. H. Sherrier, and C. E. Ravin, "Histogram-directed processing of digital chest images," Invest. Radiol., vol. 21, no. 3, pp. 253-259, 1986.

[15] _ "Implementation of adaptive filtration for digital chest imaging," Opt. Eng., vol. 26, no. 7, pp. 669-673, 1987.

[16] M. I. Sezan, A. M. Tekalp, and R. Schaetzing, "Automatic anatomically selective image enhancement in digital chest radiography," IEEE Trans. Med. Imag., vol. 8, pp. 154-162, Apr. 1989.

[17] P. M. Narendra and R. C. Fitch, "Real-time adaptive contrast enhancement," IEEE Trans. Pattern Anal. Machine Intell., vol. PAMI-3, pp. 655-661, June 1981.

[18] V. Digalakis, D. G. Manolakis, V. K. Ingle, and A. K. Kok, "Automatic adaptive contrast enhancement for radiological imaging," in Proc. 1993 IEEE Int. Symp. Circuits Syst., Chicago, IL, 1993, vol. 1, pp. 810-813.

[19] T.-L. Ji, M. K. Sundareshan, and H. Roehrig, "Adaptive image contrast enhancement based on human visual properties," IEEE Trans. Med. Imag., vol. 13, pp. 573-586, Aug. 1994.
[20] B. R. Hunt and T. M. Cannon, "Nonstationary assumption for Gaussian models of images," IEEE Trans. Syst. Man Cyber., vol. SMC-6, pp. 876-881, 1976

[21] H. T. Trussell and B. R. Hunt, "Sectioned methods for image restoration," IEEE Trans. Acoust., Speech, Signal Processing, vol. ASSP-26, pp. 157-164, 1978

[22] J. S. Milton and J. C. Arnold, Introduction to Probability and Statistics, 3rd ed. New York: McGraw-Hill, 1995.

[23] D. T. Kuan, A. A. Sawchuk, T. C. Strand, and P. Chavel, "Adaptive noise smoothing filter for images with signal-dependent noise," IEEE Trans. Pattern Anal. Machine Intell., vol. PAMI-7, pp. 165-177, 1985.

[24] R. Bernstein, "Adaptive nonlinear filters for simultaneous removal of different kinds of noise in images," IEEE Trans. Circuit Syst., vol. CS-34, pp. 1275-1291, Nov. 1987.

[25] A. Vanzo, G. Ramponi, and G. L. Sicuranza, "An image enhancement technique using polynomial filters," in Proc. 1st IEEE Int. Conf. Image Processing, Austin, TX, Nov. 1994, pp. 477-481.

[26] G. Ramponi, N. Strobel, S. K. Mitra, and T.-H. Yu, "Nonlinear unsharp masking methods for image contrast enhancement," J. Electron. Imag. vol. 5, no. 3, pp. 353-366, July 1996. 